Proc. Estonian Acad. Sci. Eng., 2002, 8, 4, 248-269

\title{
Evaluation of the Gurson-Tvergaard material model by using damage mechanics and thermomechanics
}

\author{
Kari Santaoja \\ Laboratory for Mechanics of Materials, Helsinki University of Technology, Otakaari 1, 02150 \\ Espoo, Finland; Kari.Santaoja@hut.fi \\ Received 9 October 2001, in revised form 20 February 2002

\begin{abstract}
Gurson-Tvergaard material model has been evaluated by using damage mechanics and thermomechanics. The evolution law of Tvergaard for the void volume fraction is rigorously derived. It follows that the modification for rate of the void volume fraction by Chu and Tvergaard and Tvergaard and Needleman cannot be accepted since it violates the axiom of conservation of mass. An extension of the Gurson-Tvergaard model has been derived. Using the extended model it has been shown that under compression there are stress states by which the Gurson-Tvergaard material model does not satisfy the Clausius-Duhem inequality.
\end{abstract}

Key words: material model, Gurson-Tvergaard model, porous materials, damage mechanics, thermodynamics, thermomechanics.

\section{INTRODUCTION}

The aim of the present work is the investigation of the Gurson-Tvergaard material model presented in $\left[{ }^{1,2}\right]$. This widely used constitutive equation describes the mechanical response of a porous material under stress/strain loading. Temperature effects are not included. Gurson derived his yield function by describing the mechanical response of the matrix material with a rigid-perfectly plastic constitutive equation. Although Gurson prepared yield functions for different void geometries, the model for spherical voids is referred to as the Gurson model. Tvergaard made a minor change to the yield function proposed by Gurson in order to describe the hardening of the matrix material. He then added linear elastic strain to the total deformation of the porous material. This means that his model is meant for conditions in which creep effects are negligible. Void 
nucleation and growth mechanisms with elastic-plastic material behaviour are important phenomena during ductile fracture of materials.

The aim of the present evaluation of the Gurson-Tvergaard material model is the investigation of the foundation of this model and its formulation within the thermomechanical framework. The latter will be done to verify whether the model satisfies the Clausius-Duhem inequality. Damage mechanics forms also a vital part of the analysis.

There are two widely used modifications of the original Gurson-Tvergaard material model. In the first one, Chu and Needleman $\left[{ }^{3}\right]$ added an extra term to the evolution equation of the void volume fraction $f$. According to $\left[{ }^{3}\right]$, this new term is due to the nucleation of new voids.

The second modification is developed by Tvergaard and Needleman $\left[{ }^{4}\right]$ who introduced an extra term into the model $\left[^{3}\right]$. Furthermore, in order to describe the final failure of the material, they introduced a threshold value $f_{\mathrm{C}}$. After reaching it, the void volume fraction $f$ is replaced by a new quantity $f_{\mathrm{C}}(f)$.

These modified material models are also often referred to as the GursonTvergaard material model. Thus when referring to the Gurson-Tvergaard material model one has to explain which version is meant. This study refers to the Gurson-Tvergaard material model in its original form.

\section{POROUS MATERIAL WITH LINEAR ELASTIC MATRIX}

Eshelby $\left[{ }^{5}\right]$ studied the elastic field in a Hookean material containing an ellipsoidal inclusion. As a special case, he determined the value for the complementary strain-energy density $w^{c}$ of a material containing a volume fraction $f$ of inhomogeneous spheres. For the purpose of this work the inhomogeneous spheres are "replaced" by spherical cavities. This is done by assuming that the values for the elastic constants for the cavities vanish. The spherical cavity problem was approached earlier by Mackenzie $\left[{ }^{6}\right]$, but the form of the result by Eshelby fits better the needs of this work.

The complementary strain energy density $w^{\mathrm{c}}(\boldsymbol{\sigma}, f)$ takes the form $\left[{ }^{5}\right]$

$$
w^{\mathrm{c}}(\boldsymbol{\sigma}, f)=\frac{1}{2}\left[\frac{1}{3(3 \lambda+2 \mu)}(1+A f)[\mathbf{1}: \boldsymbol{\sigma}]^{2}+\frac{1}{2 \mu}(1+B f) \mathbf{s}: \mathbf{s}\right],
$$

where $\lambda$ and $\mu$ are the Lamé constants. In Eq. (1), the stress tensor $\boldsymbol{\sigma}$ is defined as a macroscopic stress in the sense shown in Fig. 1. Stress $\sigma$ is the stress which is averaged over the entire cross-section of the body. This means that it is defined also within the voids. The microscopic stress denoted by $\tilde{\boldsymbol{\sigma}}$ is the stress between the voids and is therefore defined only outside the voids. In damage mechanics the stress tensor $\tilde{\boldsymbol{\sigma}}$ is called the effective stress tensor. The notations $\mathbf{s}$ and $\mathbf{1}$ stand for the deviatoric stress tensor and the second order identity tensor. According to Eshelby $\left[^{5}\right]$, for spherical cavities the constants $A$ and $B$ take the values 


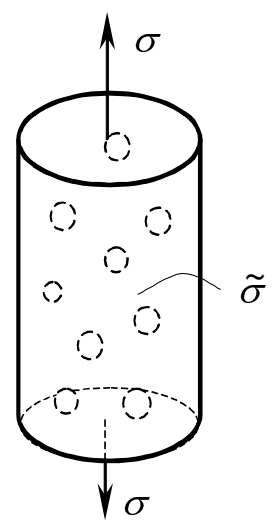

Fig. 1. A Hookean matrix material with spherical cavities.

$$
A=\frac{6 \mu+3 \lambda}{4 \mu}, \quad B=\frac{15(1-v)}{7-5 v},
$$

where $v$ is the Poisson's ratio.

According to Malvern $\left.{ }^{7}\right]$, for a fully recoverable case of isothermal deformation with reversible heat conduction the following holds:

$$
w\left(\boldsymbol{\varepsilon}^{\mathrm{e}}\right)=\rho \psi\left(\boldsymbol{\varepsilon}^{\mathrm{e}}\right),
$$

where $\boldsymbol{\varepsilon}^{\mathrm{e}}$ is the elastic strain tensor, $w\left(\boldsymbol{\varepsilon}^{\mathrm{e}}\right)$ is the strain energy density and $\psi\left(\boldsymbol{\varepsilon}^{\mathrm{e}}\right)$ is the specific Helmholtz free energy. Evidently, Eq. (3) allows the following extension:

$$
w\left(\varepsilon-\varepsilon^{\mathrm{i}}, f\right)=\rho \psi\left(\varepsilon-\varepsilon^{\mathrm{i}}, f\right),
$$

where the notation $\boldsymbol{\varepsilon}^{\mathrm{e}}:=\boldsymbol{\varepsilon}-\boldsymbol{\varepsilon}^{\mathrm{i}}$ is used. The notations $\boldsymbol{\varepsilon}$ and $\varepsilon^{i}$ stand for the strain tensor and for the inelastic strain tensor. Equation (4) also holds for the complementary functions

$$
\rho \psi^{\mathrm{c}}(\boldsymbol{\sigma}, f)=w^{\mathrm{c}}(\boldsymbol{\sigma}, f),
$$

where the specific complementary Helmholtz free energy $\psi^{\mathrm{c}}(\boldsymbol{\sigma}, f)$ and the stress tensor $\boldsymbol{\sigma}$ in the initial configuration are assumed to vanish.

From Eqs. (1) and (5), we obtain

$$
\psi^{\mathrm{c}}(\boldsymbol{\sigma}, f)=\frac{1}{2 \rho}\left[\frac{1}{3(3 \lambda+2 \mu)}(1+A f)[\mathbf{1}: \boldsymbol{\sigma}]^{2}+\frac{1}{2 \mu}(1+B f) \mathbf{s}: \mathbf{s}\right] .
$$

According to $\left[{ }^{8}\right]$, for the present set of state variables the state equations are

$$
\begin{gathered}
\boldsymbol{\varepsilon}-\boldsymbol{\varepsilon}^{\mathrm{i}}=\rho \frac{\partial \psi^{\mathrm{c}}(\boldsymbol{\sigma}, f)}{\partial \boldsymbol{\sigma}}, \\
g=\rho \frac{\partial \psi^{\mathrm{c}}(\boldsymbol{\sigma}, f)}{\partial f} .
\end{gathered}
$$

In Eq. (7b) the notation $g$ stands for the internal force associated with the void volume fraction $f$. Substitution of Eq. (6) into Eq. (7a) gives

$$
\boldsymbol{\varepsilon}-\boldsymbol{\varepsilon}^{\mathrm{i}}=\frac{1}{3(3 \lambda+2 \mu)}(1+A f) \mathbf{1}: \boldsymbol{\sigma} \mathbf{1}+\frac{1}{2 \mu}(1+B f) \mathbf{s} .
$$

In deriving Eq. (8), the following equalities were used: 


$$
\frac{\partial(\mathbf{1}: \boldsymbol{\sigma})}{\partial \boldsymbol{\sigma}}=\mathbf{1}: \frac{\partial \boldsymbol{\sigma}}{\partial \boldsymbol{\sigma}}=\mathbf{1}: \mathbf{I}=\mathbf{1}, \quad \frac{\partial}{\partial \boldsymbol{\sigma}}(\mathbf{s}: \mathbf{s})=2 \mathbf{s},
$$

where the deviatoric stress tensor $\mathbf{s}$ is defined as

$$
\mathbf{s}:=\mathbf{K}: \boldsymbol{\sigma},
$$

where

$$
\mathbf{K}:=\mathbf{I}-\frac{1}{3} \mathbf{1} \mathbf{1}
$$

Notation I stands for the fourth order identity tensor.

Using Eq. (10a), Eq. (8) can be written as

$$
\boldsymbol{\varepsilon}-\boldsymbol{\varepsilon}^{\mathrm{i}}=\frac{1}{3(3 \lambda+2 \mu)}(1+A f) \mathbf{1} \mathbf{1}: \boldsymbol{\sigma}+\frac{1}{2 \mu}(1+B f) \mathbf{K}: \boldsymbol{\sigma},
$$

or

$$
\boldsymbol{\varepsilon}-\boldsymbol{\varepsilon}^{\mathrm{i}}=\tilde{\mathbf{S}}(f): \boldsymbol{\sigma},
$$

where $\tilde{\mathbf{S}}(f)$ is the fourth order compliance tensor for a Hookean material with spherical voids. It is defined by

$$
\widetilde{\mathbf{S}}(f):=\frac{1}{3(3 \lambda+2 \mu)}(1+A f) \mathbf{1} \mathbf{1}+\frac{1}{2 \mu}(1+B f) \mathbf{K} .
$$

The compliance tensor $\tilde{\mathbf{S}}(f)$ can be divided into two parts:

$$
\tilde{\mathbf{S}}(f):=\mathbf{S}+\mathbf{S}^{\mathrm{v}}(f),
$$

where the compliance tensors $\mathbf{S}$ and $\mathbf{S}^{\mathrm{v}}(f)$ are defined as

$$
\mathbf{S}:=\frac{1}{3(3 \lambda+2 \mu)} \mathbf{1 1}+\frac{1}{2 \mu} \mathbf{K},
$$

and

$$
\mathbf{S}^{\mathrm{v}}(f):=\frac{A f}{3(3 \lambda+2 \mu)} \mathbf{1 1}+\frac{B f}{2 \mu} \mathbf{K} .
$$

Substitution of Eq. (6) into Eq. (7b) yields

$$
g=\frac{1}{2}\left[\frac{A}{3(3 \lambda+2 \mu)}[\mathbf{1}: \boldsymbol{\sigma}]^{2}+\frac{B}{2 \mu} \mathbf{s}: \mathbf{s}\right] \text {. }
$$




\section{EFFECTIVE STRESS TENSOR}

According to Chaboche $\left[{ }^{9}\right]$, the constitutive equation for the damaged Hookean material can be expressed as

$$
\begin{gathered}
\boldsymbol{\sigma}=\tilde{\mathbf{C}}(f): \boldsymbol{\varepsilon}^{\mathrm{e}}, \\
\tilde{\boldsymbol{\sigma}}:=\mathbf{C}: \boldsymbol{\varepsilon}^{\mathrm{e}},
\end{gathered}
$$

where $\tilde{\mathbf{C}}(f)$ is the fourth order constitutive tensor for a damaged Hookean material. The above model can also be found in the paper by Lemaitre and Chaboche $\left[{ }^{10}\right]$ although this source contains a misprint. The author has added the variable $f$ to the constitutive tensor $\widetilde{\mathbf{C}}(f)$.

According to $\left[{ }^{10}\right]$, the effective stress tensor $\tilde{\boldsymbol{\sigma}}$ is the stress calculated over the section (of the damaged material) which effectively resists the forces. The same interpretation for the effective stress tensor $\tilde{\boldsymbol{\sigma}}$ can be found in papers by Rabotnov $\left[{ }^{11}\right]$, Hult $\left[{ }^{12}\right]$, and Santaoja $\left[{ }^{13}\right]$. Santaoja $\left[{ }^{14}\right]$ made a simple uniaxial tube/bar investigation to show the above interpretation for the effective stress tensor $\tilde{\boldsymbol{\sigma}}$.

Thus in the material, modelled in this work, the effective stress tensor $\tilde{\boldsymbol{\sigma}}$ describes the stress in the matrix material between the voids. It is of course a homogenized stress over the representative volume $V^{\text {rep }}$ of the damaged material.

The inverses of the constitutive tensors $\mathbf{C}$ and $\widetilde{\mathbf{C}}(f)$ are denoted by $\mathbf{C}^{-1}$ or $\mathbf{S}$ and $\widetilde{\mathbf{C}}^{-1}(f)$ or $\widetilde{\mathbf{S}}(f)$, respectively. Multiplying Eq. (18a) from the left by the tensor $\tilde{\mathbf{S}}(f)$ yields

$$
\boldsymbol{\varepsilon}^{\mathrm{e}}=\widetilde{\mathbf{S}}(f): \boldsymbol{\sigma},
$$

which is identical to Eq. (12). Substitution of Eq. (19) into Eq. (18b) gives

$$
\tilde{\boldsymbol{\sigma}}:=\mathbf{C}: \widetilde{\mathbf{S}}(f): \boldsymbol{\sigma} .
$$

\section{GURSON-TVERGAARD MATERIAL MODEL}

In Gurson's constitutive equation for modelling pressure-dependent plastic deformation of metallic materials $\left[{ }^{1}\right]$, pressure dependence is due to void nucleation and growth. Gurson evaluated a unit cube of porous material, the void volume fraction of which was denoted by the variable $f$. He assumed that the matrix material, i.e., the material between the voids, can be approximated by the rigid-perfectly plastic material model. Gurson used the von Mises yield criterion in determining the onset of plastic yield in the matrix material.

In his evaluation Gurson used two types of variables - macroscopic and microscopic. He studied a small piece of porous material which he called "a unit cube of porous material of volume $V$, large enough to be statistically 
representative of the properties of the aggregate". The term unit cube is here replaced by the term representative volume, denoted by $V^{\text {rep }}$. The representative volume of the material $V^{\text {rep }}$ is large enough to be used in homogenization of microscale effects in the macroscale material, yet small enough to provide values for continuous field variables at a given point in a structure. In a macroscopic material model, the real structure of the material in the representative volume $V^{\text {rep }}$ is averaged over this volume, throughout which the macroscopic field variables have the same values. Values of the microscopic quantities, however, vary realistically within $V^{\text {rep }}$. In the following text the terms variable, quantity, etc. apply to the macroscopic phenomena. Only in cases in which the author wishes to stress the difference between macroscopic and microscopic quantities is the term macroscopic used.

By using the upper bound approach, Gurson derived macroscopic yield functions for materials having different shapes of voids. The result for spherical voids with fully plastic flow of the matrix material is referred to as the Gurson model. The yield function for the Gurson model is $\left[{ }^{1}\right]$

$$
F(\boldsymbol{\sigma}, f)=\left\{\frac{J_{\mathrm{vM}}(\boldsymbol{\sigma})}{\sigma^{\mathrm{Y}}}\right\}^{2}+2 f \cosh \left(\frac{\mathbf{1 : \boldsymbol { \sigma }}}{2 \sigma^{\mathrm{Y}}}\right)-1-f^{2},
$$

where $J_{\mathrm{vM}}(\cdot)$ is the von Mises operator and $\sigma^{\mathrm{Y}}$ is the microscopic equivalent tensile yield stress. According to $\left[{ }^{1}\right]$, the quantity $\sigma^{\mathrm{Y}}$ is the uniaxial yield strength of the matrix material. The parameter $\sigma^{\mathrm{Y}}$ has a fixed value. In Eq. (21), the variables $\boldsymbol{\sigma}$ and $f$ are macroscopic, which means that they describe the response of the representative volume $V^{\text {rep }}$; i.e., they are variables of the ideal material where the behaviour of the voids and matrix material are homogenized within the representative volume $V^{\text {rep }}$. In the appendix of his paper Gurson showed that the normality rule holds also on the macroscopic level. Therefore the normality rule derived in the previous pages of this work can be used with Eq. (21).

Some years later Tvergaard $\left[{ }^{2}\right]$ investigated the influence of voids on shear band instabilities. He studied the problem numerically using two different approaches. First, he performed a finite element analysis using a fine microscale mesh for description of an elastic-plastic medium containing a double periodic array of circular cylindrical voids. Second, he used a continuum model with a macroscopic material model for evaluation of the same problem. For his continuum model analysis, Tvergaard modified Gurson's yield function (21) by introducing three new material parameters and assuming that the matrix material shows strain hardening. The latter means that Tvergaard replaced the constant $\sigma^{\mathrm{Y}}$ in Eq. (21) with a variable (describing hardening) denoted by $\sigma^{\mathrm{M}}$. According to Tvergaard, $\sigma^{\mathrm{M}}$ represents the equivalent tensile flow stress in the matrix material, disregarding local stress variations. Tvergaard $\left[{ }^{2}\right]$ introduced the following yield function: 


$$
F\left(\boldsymbol{\sigma}, \sigma^{\mathrm{M}}, f\right)=\left\{\frac{J_{\mathrm{vM}}(\boldsymbol{\sigma})}{\sigma^{\mathrm{M}}}\right\}^{2}+2 f q^{1} \cosh \left(\frac{q^{2} \mathbf{1}: \boldsymbol{\sigma}}{2 \sigma^{\mathrm{M}}}\right)-\left(1+q^{3} f^{2}\right) .
$$

He further added to the model elastic deformation, which he assumed to obey Hooke's law.

Tvergaard introduced his modification in order to obtain a better fit between the numerical results of his microscopic finite element analysis and the macroscopic continuum model $\left[^{2}\right]$. By assuming that the constants $q^{1}=q^{2}=q^{3} \equiv 1$, that the variable $\sigma^{\mathrm{M}}$ takes a constant value $\sigma^{\mathrm{Y}}$, and that the elastic deformation can be neglected as a small quantity, the Gurson-Tvergaard material model reduces to the Gurson model.

Elastic deformation is described by Hooke's law which can be written as

$$
\boldsymbol{\sigma}=\mathbf{C}: \boldsymbol{\varepsilon}^{\mathrm{e}},
$$

where the fourth order constitutive tensor $\mathbf{C}$ is defined by

$$
\mathbf{C}:=\lambda \mathbf{1 1}+2 \mu \mathbf{I}^{\mathrm{s}} \text {. }
$$

Here $\lambda$ and $\mu$ are Lamé constants defined by

$$
\lambda:=\frac{v E}{(1+v)(1-2 v)}, \quad \mu:=\frac{E}{2(1+v)} .
$$

In Eqs. (25) $E$ is Young's modulus. The fourth-order symmetric identity tensor $\mathbf{I}^{\mathrm{S}}$ is defined by

$$
\mathbf{I}^{\mathrm{s}}:=\frac{1}{2}\left(\delta_{i k} \delta_{j l}+\delta_{i l} \delta_{j k}\right) \vec{i}_{i} \vec{i}_{j} \vec{i}_{k} \vec{i}_{l} .
$$

The evolution equations for the quantity $\sigma^{\mathrm{M}}$, for the void volume fraction $f$, and for the plastic strain $\boldsymbol{\varepsilon}^{\mathrm{p}}$ are derived next. Although Eq. (21), proposed by Gurson, contained the void volume fraction $f$ as a variable, he did not give any evolution equation for it. The expressions for $\dot{\sigma}^{\mathrm{M}}$ and $\dot{f}$ have been derived by Tvergaard $\left[{ }^{2}\right]$.

\subsection{Evolution equation for the quantity $\sigma^{\mathrm{M}}$}

The (classical) plasticity theory (see, e.g., Santaoja $\left[{ }^{15}\right]$ ) derives the expression for $\dot{\sigma}^{\mathrm{M}}$ as follows. A uniaxial stress-strain curve is drawn for elastic-plastic material behaviour (Fig. 2). In this work the matrix material is assumed to behave accordingly. The uniaxial strain terms of the matrix material are denoted by $\varepsilon^{\mathrm{m}}$ (total strain), $\varepsilon^{\mathrm{me}}$ (elastic strain) and $\varepsilon^{\mathrm{mp}}$ (plastic strain). The uniaxial yield stress of the matrix material is denoted by $\sigma^{\text {my }}$. The value of $\sigma^{\text {my }}$ varies with hardening. The notations $E^{\mathrm{m}}$ and $E^{\mathrm{mp}}$ in Fig. 2 refer to the Young's modulus 


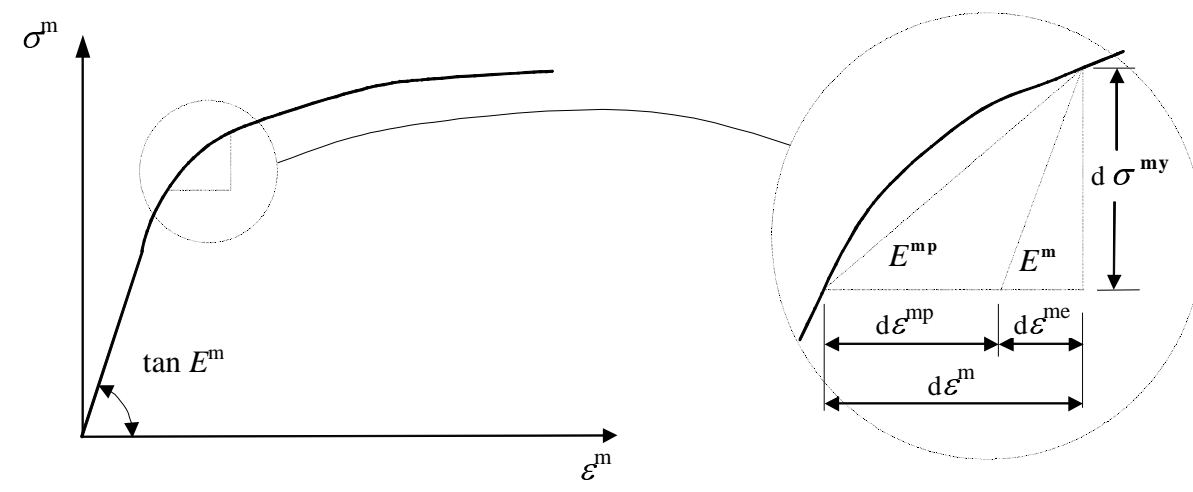

Fig. 2. Uniaxial stress-strain curve for an elastic-plastic (matrix) material behaviour.

and the slope of the uniaxial stress-strain curve beyond the elastic region, i.e. to the tangent modulus of the matrix material, respectively.

Figure 2 gives

$$
\mathrm{d} \sigma^{\mathrm{my}}=E^{\mathrm{mp}} \mathrm{d} \varepsilon^{\mathrm{m}},
$$

which yields

$$
\mathrm{d} \varepsilon^{\mathrm{m}}=\frac{\mathrm{d} \sigma^{\mathrm{my}}}{E^{\mathrm{mp}}},
$$

and

$$
\mathrm{d} \sigma^{\mathrm{my}}=E^{\mathrm{m}} \mathrm{d} \varepsilon^{\mathrm{me}},
$$

which yields

$$
\mathrm{d} \varepsilon^{\mathrm{me}}=\frac{\mathrm{d} \sigma^{\mathrm{my}}}{E^{\mathrm{m}}} .
$$

From Fig. 2 and Eqs. (27b) and (28b) follows

$$
\mathrm{d} \varepsilon^{\mathrm{mp}}=\mathrm{d} \varepsilon^{\mathrm{m}}-\mathrm{d} \varepsilon^{\mathrm{me}}=\left(\frac{1}{E^{\mathrm{mp}}}-\frac{1}{E^{\mathrm{m}}}\right) \mathrm{d} \sigma^{\mathrm{my}},
$$

which can be written as

$$
\dot{\varepsilon}^{\mathrm{mp}}=\left(\frac{1}{E^{\mathrm{mp}}}-\frac{1}{E^{\mathrm{m}}}\right) \dot{\sigma}^{\mathrm{my}} .
$$

Figure 2 requires the following comments. Young's modulus $E^{\mathrm{m}}$ and the slope of the uniaxial stress-strain curve beyond the elastic region $E^{\mathrm{mp}}$ are expressed in the extended picture on the right-hand side of Fig. 2. The reader may notice that the angles are $\tan E^{\mathrm{m}}$ and $\tan E^{\mathrm{mp}}$. This is expressed in the stress-strain curve on the left-hand side of Fig. 2. Furthermore, $E^{\mathrm{mp}}$ is the 
tangent modulus but drawn as a "semi-secant modulus" in Fig. 2. However, the picture within the circle is infinitesimal. For infinitesimal quantities the "semisecant modulus" and the "tangent modulus" coincide. For rate equations, such as Eq. (30), the difference vanishes completely.

According to Tvergaard $\left[^{2}\right]$, at plastic loading the increment of the effective plastic strain $\varepsilon^{\mathrm{Mp}}$ in the matrix material with current tangent modulus $E^{\mathrm{t}}$ is

$$
\dot{\varepsilon}^{\mathrm{Mp}}=\left(\frac{1}{E^{\mathrm{t}}}-\frac{1}{E}\right) \dot{\sigma}^{\mathrm{M}}
$$

Assuming that $\varepsilon^{\mathrm{Mp}}$ varies according to the equivalent plastic work expression

$$
\boldsymbol{\sigma}: \dot{\boldsymbol{\varepsilon}}^{\mathrm{pv}}=(1-f) \sigma^{\mathrm{M}} \dot{\varepsilon}^{\mathrm{Mp}},
$$

the increment of $\sigma^{\mathrm{M}}$ is given by

$$
\dot{\sigma}^{\mathrm{M}}=\frac{E E^{\mathrm{t}}}{E-E^{\mathrm{t}}} \frac{\boldsymbol{\sigma}: \dot{\boldsymbol{\varepsilon}}^{\mathrm{pv}}}{(1-f) \sigma^{\mathrm{M}}} .
$$

The above expressions by Tvergaard raise several questions. The first one is related to terminology. Tvergaard calls macroscopic deformation, which is due to (traditional) plastic flow and to void nucleation and growth, "plastic strain", and gives to it the superscript " $p$ " $\left[{ }^{2}\right]$. In order to show the difference between pure plastic flow and the plasticity of porous materials, the author has in Eqs. (32) and (33) used notation $\boldsymbol{\varepsilon}^{\mathrm{pv}}$ and has called it the "void-plastic strain tensor".

According to Tvergaard $\left[{ }^{2}\right]$, the quantity $\sigma^{\mathrm{M}}$ represents the equivalent tensile flow stress in the matrix material, disregarding local stress variations. Thus, $\sigma^{\mathrm{M}}$ is a "semi-macroscopic" or mesoscopic variable. It is obtained by reducing the multiaxial stress field to a uniaxial one (e.g. by using the von Mises equivalent stress concept) and averaging it over the matrix material within the representative volume $V^{\text {rep }}$. The (real) macroscopic variables are averaged over the entire volume of $V^{\text {rep }}$. Figure 3 explains the difference between the terms "microscopic", "mesoscopic" (semi-macroscopic) and "macroscopic". The capital superscript M refers to mesoscopic.

In order to make some sense of Eq. (31), the quantities $\dot{\varepsilon}^{\mathrm{mp}}$ and $E^{\mathrm{t}}$ must also be mesoscopic. The derivation of Eq. (31) is missing in $\left[{ }^{2}\right]$. As the author believes that Eq. (30) inspired Tvergaard to write Eq. (31), the latter must therefore be interpreted as a model. One problem is determination of the value for the current tangent modulus $E^{\mathrm{t}}$. Since it is a semi-macroscopic variable, it should be a kind of an integral of the microscopic Young's moduli over the matrix material in the representative volume $V^{\text {rep }}$. Since no stress-strain curve for mesoscopic material behaviour is available, researchers may use the microscopic stress-strain relation (sketched in Fig. 2) and they may replace the microscopic stress $\sigma^{\mathrm{m}}$ by $\sigma^{\mathrm{M}}$ and the microscopic strain $\varepsilon^{\mathrm{m}}$ by $\varepsilon^{\mathrm{M}}$. Thus in practice the axes are mesoscopic but the stress-strain curve is a microscopic one. 


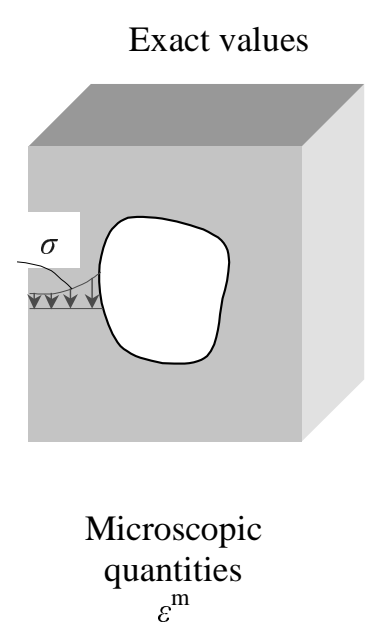

(a)
Uniaxialised and averaged values (outside voids)

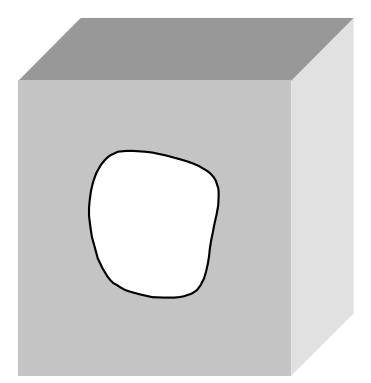

Mesoscopic quantities $\varepsilon^{\mathrm{M}}$

(b)
Averaged values (over the entire $V^{\text {rep }}$ )

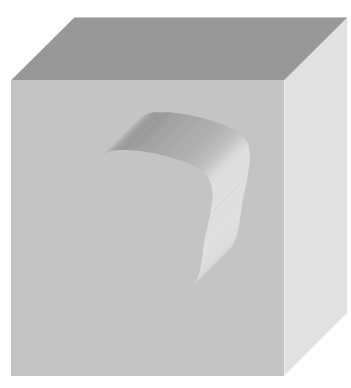

Macroscopic
quantities

(c)

Fig. 3. (a) Values for the microscopic quantities vary within the matrix material; they are not defined in voids; (b) semi-macroscopic quantities are obtained by averaging the microscopic values and by reducing the nine tensor components into one scalar-valued variable; (c) macroscopic quantities are averaged over the entire volume of the representative volume $V^{\text {rep }}$.

In the "traditional" theory of plasticity, when isotropic hardening is modelled, two different concepts are often used [ $\left.{ }^{7}\right]$. In the following the term "microscopic" is adopted to show that the quantities presented are microscopic ones in the sense of this work. The next simplest (after perfect plasticity) hardening assumption that the yield surface maintains its shape, while its size increase is controlled by a single parameter depending on the plastic deformation - is called isotropic hardening. It remains only to specify the size-determining parameter and its dependence on deformation. Two different schemes have been used for this, which are different in general but reduce to the same when used appropriately with the von Mises yield condition.

The first one is the idea of a universal plastic stress-strain curve. With this concept it is assumed that there exists a universal plastic stress-strain curve relating two scalar quantities - the (microscopic) effective stress $\sigma_{\text {eff }}^{\mathrm{m}}$ (measuring the size of the yield surface) and the integral of the (microscopic) effective plastic strain increment $\mathrm{d} \varepsilon_{\text {eff }}^{\mathrm{mp}}$. Thus the universal plastic stress-strain curve is defined by

$$
\sigma_{\text {eff }}^{\mathrm{m}}=H\left(\int \mathrm{d} \varepsilon_{\mathrm{eff}}^{\mathrm{mp}}\right)
$$

When the von Mises yield condition is used, Eq. (34) takes the form

$$
J_{\mathrm{vM}}\left(\boldsymbol{\sigma}^{\mathrm{m}}\right)=H\left(\int \mathrm{d} J_{\mathrm{vM}}\left(\boldsymbol{\varepsilon}^{\mathrm{mp}}\right)\right) \text {. }
$$


Energy condition for strain hardening. The second concept is that the size of the yield surface depends only on the (microscopic) total plastic work $W^{\mathrm{mp}}$. This means that the (microscopic) effective stress $\sigma_{\text {eff }}^{\mathrm{m}}$ is a single-valued function of (microscopic) total plastic work $W^{\mathrm{mp}}$. It can be written as

$$
\sigma_{\text {eff }}^{\mathrm{m}}=F\left(W^{\mathrm{mp}}\right),
$$

where

$$
W^{\mathrm{mp}}:=\int \boldsymbol{\sigma}^{\mathrm{m}}: \mathrm{d} \boldsymbol{\varepsilon}^{\mathrm{mp}}
$$

When the von Mises yield function is used, the following is obtained $\left[^{7}\right]$ :

$$
W^{\mathrm{mp}}:=\int J_{\mathrm{vM}}\left(\boldsymbol{\sigma}^{\mathrm{m}}\right) \mathrm{d} J_{\mathrm{vM}}\left(\boldsymbol{\varepsilon}^{\mathrm{mp}}\right) .
$$

Equations (37) and (38) give

$$
\boldsymbol{\sigma}^{\mathrm{m}}: \mathrm{d} \boldsymbol{\varepsilon}^{\mathrm{mp}}=J_{\mathrm{vM}}\left(\boldsymbol{\sigma}^{\mathrm{m}}\right) \mathrm{d} J_{\mathrm{vM}}\left(\boldsymbol{\varepsilon}^{\mathrm{mp}}\right),
$$

or

$$
\boldsymbol{\sigma}^{\mathrm{m}}: \dot{\boldsymbol{\varepsilon}}^{\mathrm{mp}}=J_{\mathrm{vM}}\left(\boldsymbol{\sigma}^{\mathrm{m}}\right) J_{\mathrm{vM}}\left(\dot{\boldsymbol{\varepsilon}}^{\mathrm{mp}}\right) .
$$

In writing Eq. (32), Tvergaard may have integrated Eq. (40) "formally" over the representative volume $V^{\text {rep }}$. The term $(1-f)$ on the right-hand side of Eq. (32) originates from the fact that the quantities $\sigma^{\mathrm{M}}$ and $\dot{\varepsilon}^{\mathrm{M}}$ are not defined in the voids where the macroscopic quantities $\boldsymbol{\sigma}$ and $\dot{\boldsymbol{\varepsilon}}^{\mathrm{pv}}$ have a certain value.

According to Eq. (38), when the von Mises yield condition is used, the (microscopic) plastic work $W^{\mathrm{mp}}$ equals the area under the universal plastic stress-strain curve defined by the von Mises value of the (microscopic) stress $J_{\mathrm{vM}}\left(\boldsymbol{\sigma}^{\mathrm{m}}\right)$ and the von Mises value of the (microscopic) plastic strain increment $\mathrm{d} J_{\mathrm{VM}}\left(\boldsymbol{\varepsilon}^{\mathrm{mp}}\right)$. In the uniaxial tension/compression, the (microscopic) stress $\boldsymbol{\sigma}^{\mathrm{m}}$ coincides with the von Mises value of the (microscopic) stress $J_{\mathrm{vM}}\left(\boldsymbol{\sigma}^{\mathrm{m}}\right)$. The same holds for the strains. This means that the uniaxial plastic stress-strain curve can be interpreted as the universal plastic stress-strain curve. The former is then connected to the multiaxial quantities through Eq. (39), which is widely used in the traditional theory of plasticity (see, e.g., Santaoja $\left[{ }^{15}\right]$ or Zienkiewicz $\left[{ }^{16}\right]$ ).

The Odqvist parameter $O$ is defined as

$$
O=\int_{0}^{t} J_{\mathrm{vM}}\left(\dot{\boldsymbol{\varepsilon}}^{\mathrm{mp}}\right) \mathrm{d} t=\int_{0}^{t}\left[\frac{3}{2} \dot{\boldsymbol{\varepsilon}}^{\mathrm{mp}}: \dot{\boldsymbol{\varepsilon}}^{\mathrm{mp}}\right]^{1 / 2} \mathrm{~d} t .
$$

It can be used as a measure of isotropic hardening $\left[{ }^{17}\right]$. 


\subsection{Evolution equation for porosity $f$}

Tvergaard has stated that as the matrix material is assumed to be plastically incompressible, the increment of the void volume fraction is given by $\left[{ }^{2}\right]$

$$
\dot{f}=(1-f) \mathbf{1}: \dot{\boldsymbol{\varepsilon}}^{\mathrm{pv}} .
$$

In Eq. (42), $\boldsymbol{\varepsilon}^{\mathrm{pv}}$ is the void-plastic strain tensor. It models the plastic strain of the matrix material and the strain due to porosity change. The notation $\boldsymbol{\varepsilon}^{\mathrm{pv}}$ and the name "void-plastic strain tensor" are introduced by the author of the present paper.

Comments to the above presentation are as follows. According to Narasimhan $\left[{ }^{18}\right]$, the material derivative of the representative volume $\dot{V}^{\text {rep }}$ takes the form

$$
\dot{V}^{\text {rep }}=(\vec{\nabla} \cdot \vec{v}) V^{\text {rep }} .
$$

In $\left[{ }^{18}\right]$ the relationship between the divergence of the velocity vector $\vec{\nabla} \cdot \vec{v}$ and the rate of deformation tensor $\mathbf{d}$ is obtained as

$$
\vec{\nabla} \cdot \vec{v}=\mathbf{1}: \mathbf{d} .
$$

For small displacement gradients and small velocity gradients the rate of the deformation tensor $\mathbf{d}$ and the strain rate tensor $\dot{\boldsymbol{\varepsilon}}$ coincide

$$
\mathbf{d}=\dot{\boldsymbol{\varepsilon}}
$$

Combining Eqs. (43)-(45) gives

$$
\dot{V}^{\text {rep }}=\mathbf{1}: \dot{\boldsymbol{\varepsilon}} V^{\text {rep }} .
$$

The volume of the matrix material within the representative volume $V_{\text {mat }}^{\text {rep }}$ can be expressed as

$$
V_{\text {mat }}^{\text {rep }}=(1-f) V^{\text {rep }},
$$

which leads to

$$
\dot{V}_{\text {mat }}^{\text {rep }}=(1-f) \dot{V}^{\text {rep }}-\dot{f} V^{\text {rep }} .
$$

By substituting Eq. (46) into (48), we obtain

$$
\dot{V}_{\text {mat }}^{\text {rep }}=(1-f) \mathbf{1}: \dot{\boldsymbol{\varepsilon}} V^{\text {rep }}-\dot{f} V^{\text {rep }},
$$

which gives

$$
\dot{f}=(1-f) \mathbf{1}: \dot{\boldsymbol{\varepsilon}}-\frac{\dot{V}_{\mathrm{mat}}^{\text {rep }}}{V^{\text {rep }}} .
$$


The (total) macroscopic strain tensor $\boldsymbol{\varepsilon}$ can be assumed to be separable as follows:

$$
\boldsymbol{\varepsilon}=\boldsymbol{\varepsilon}^{\mathrm{e}}+\boldsymbol{\varepsilon}^{\mathrm{p}}+\boldsymbol{\varepsilon}^{\mathrm{v}},
$$

where $\boldsymbol{\varepsilon}^{\mathrm{e}}$ is the elastic deformation (due to the matrix material and void), $\boldsymbol{\varepsilon}^{\mathrm{p}}$ is the plastic deformation (due to the matrix material), and $\boldsymbol{\varepsilon}^{\mathrm{v}}$ is the deformation due to void nucleation and growth. The latter two strain tensors can be added together and therefore the following expression is valid

$$
\boldsymbol{\varepsilon}^{\mathrm{pv}}=\boldsymbol{\varepsilon}^{\mathrm{p}}+\boldsymbol{\varepsilon}^{\mathrm{v}}
$$

where $\varepsilon^{\mathrm{pv}}$ is the void-plastic strain tensor introduced above.

The plastic deformation of the matrix material does not cause any volume changes. The elastic deformation of the matrix material can be assumed to be very small and therefore the volume change due to elastic deformation can be neglected as a small quantity. This means that the term $\dot{V}_{\text {mat }}^{\text {rep }} / V^{\text {rep }}$ can be assumed to vanish, which implies that the quantity $\mathbf{1}: \dot{\boldsymbol{\varepsilon}}$ in Eq. (50) is due to void nucleation and growth. Thus Eq. (50) gives the following result:

$$
\dot{f}=(1-f) \mathbf{1}: \dot{\boldsymbol{\varepsilon}}^{\mathrm{v}} .
$$

Since pure plastic flow does not contain any dilatation, the strain rate measures 1: $\dot{\boldsymbol{\varepsilon}}^{\mathrm{pv}}$ and 1: $\dot{\boldsymbol{\varepsilon}}^{\mathrm{v}}$ coincide. Therefore Eq. (53) can be written as

$$
\dot{f}=(1-f) \mathbf{1}: \dot{\boldsymbol{\varepsilon}}^{\mathrm{pv}} .
$$

Equation (54) has the same form as Eq. (42) that was the aim of this part of the paper.

Chu and Needleman $\left[{ }^{3}\right]$ were not satisfied with Eq. (54) but replaced it by the following:

$$
\dot{f}=\dot{f}_{\text {growth }}+\dot{f}_{\text {nucleation }},
$$

where the first term is related to the growth of the existing voids and the second one is related to the increase of void volume fraction due to the nucleation of new voids. The first term is

$$
\dot{f}_{\text {growth }}=(1-f) \mathbf{1}: \dot{\boldsymbol{\varepsilon}}^{\mathrm{pv}} .
$$

Chu and Needleman $\left[{ }^{3}\right]$ gave three expressions for the term $\dot{f}_{\text {nucleation }}$. They are not given here, since the above derivation by the author shows no extra term beyond the right-hand side of Eq. (54) and they cannot be added to the evolution equation of the void volume fraction $f$. Those extra terms violate the axiom of conservation of mass and therefore are to be excluded.

Tvergaard and Needleman $\left[{ }^{4}\right]$ adopted Eqs. (55) and (56). Furthermore, they introduced a new modification for $f$ by replacing it in the yield function $F$ by the quantity $f *$ which is defined as 


$$
f^{*}:=\left\{\begin{array}{cc}
f & \text { when } f \leq f_{\mathrm{C}}, \\
f_{\mathrm{C}}+K\left(f-f_{\mathrm{C}}\right) & \text { when } x \geq 0 .
\end{array}\right.
$$

In Eq. (57) $K$ is a constant related to the ultimate value of $f^{*}$ at which the macroscopic stress-carrying capacity vanishes.

As mentioned above, the evolution equation of the void volume fraction $f$ cannot be derived from Eq. (54), since the axiom of conservation of mass is violated. Equation (57) models the descending part of the stress-strain relation, i.e. strain softening. In finite element analysis, strain softening causes localization and thus influences reliability of the computed results.

\subsection{Evolution equation for the void-plastic strain tensor $\varepsilon^{p v}$}

Derivation of the evolution equation by Tvergaard $\left[{ }^{2}\right]$, with notations of the author, is as follows. When the yield function $F$ is known, the normality rule provides the expression for the void-plastic strain rate tensor $\dot{\boldsymbol{\varepsilon}}^{\mathrm{pv}}$

$$
\dot{\boldsymbol{\varepsilon}}^{\mathrm{pv}}=\stackrel{\circ}{\lambda} \frac{\partial F\left(\boldsymbol{\sigma}, \sigma^{\mathrm{M}}, f\right)}{\partial \boldsymbol{\sigma}} .
$$

The value for the plasticity multiplier $\dot{\lambda}$ is obtained from the consistency condition

$$
\dot{F}=0 \text {. }
$$

Tvergaard has written the following $\left[{ }^{2}\right]$

$$
\dot{\boldsymbol{\varepsilon}}^{\mathrm{pv}}=\frac{1}{H}\left[\frac{3}{2} \frac{\mathbf{s}}{\sigma^{\mathrm{M}}}+a \mathbf{1}\right]\left[\frac{3}{2} \frac{\mathbf{s}}{\sigma^{\mathrm{M}}}+a \mathbf{1}\right]: \boldsymbol{\sigma}
$$

where

$$
a=\frac{f q^{1} q^{2}}{2} \sinh \left(\frac{q^{2} \mathbf{1}: \boldsymbol{\sigma}}{2 \sigma^{\mathrm{M}}}\right),
$$

and

$$
\begin{aligned}
H= & \frac{E E^{\mathrm{t}}}{\left(E-E^{\mathrm{t}}\right)(1-f)}\left[\left\{\frac{J_{\mathrm{vM}}(\boldsymbol{\sigma})}{\sigma^{\mathrm{M}}}\right\}^{2}+a \frac{\mathbf{1}: \boldsymbol{\sigma}}{\sigma^{\mathrm{M}}}\right]^{2} \\
& -3 \sigma^{\mathrm{M}} a(1-f)\left[q^{1} \cosh \left(\frac{q^{2} \mathbf{1}: \boldsymbol{\sigma}}{2 \sigma^{\mathrm{M}}}\right)-q^{3} f\right] .
\end{aligned}
$$

Since the expressions of the author have strongly different appearances compared with the corresponding expressions by Tvegaard $\left[{ }^{2}\right]$, we proceed as follows. 
Yield function of Tvergaard is $\left[{ }^{2}\right]$

$$
F\left(\boldsymbol{\sigma}, \sigma^{\mathrm{M}}, f\right)=\left\{\frac{J_{\mathrm{vM}}(\boldsymbol{\sigma})}{\sigma^{\mathrm{M}}}\right\}^{2}+2 f q^{1} \cosh \left(\frac{q^{2} \mathbf{1}: \boldsymbol{\sigma}}{2 \sigma^{\mathrm{M}}}\right)-\left(1+q^{3} f^{2}\right) .
$$

Applying the normality rule of plasticity with the associated flow rule (see, e.g. $\left.\left[{ }^{19}\right]\right)$, Eq. (63) provides the following rate equation:

$$
\dot{\boldsymbol{\varepsilon}}^{\mathrm{pv}}=\stackrel{\circ}{\lambda} \frac{\partial F\left(\boldsymbol{\sigma}, \sigma^{\mathrm{M}}, f\right)}{\partial \boldsymbol{\sigma}}=\stackrel{\circ}{\lambda}\left\{\frac{3 \mathbf{s}}{\left(\sigma^{\mathrm{M}}\right)^{2}}+\frac{f q^{1} q^{2}}{\sigma^{\mathrm{M}}} \sinh \left(\frac{q^{2} \mathbf{1}: \boldsymbol{\sigma}}{2 \sigma^{\mathrm{M}}}\right) \mathbf{1}\right\} .
$$

By deriving Eq. (64), the following equalities were used:

$$
\frac{\partial J_{\mathrm{vM}}(\boldsymbol{\sigma})}{\partial \boldsymbol{\sigma}}=\frac{3}{2} \frac{\mathbf{s}}{J_{\mathrm{vM}}(\boldsymbol{\sigma})}, \quad \frac{\partial(\mathbf{1}: \boldsymbol{\sigma})}{\partial \boldsymbol{\sigma}}=\mathbf{1}: \frac{\partial \boldsymbol{\sigma}}{\partial \boldsymbol{\sigma}}=\mathbf{1}: \mathbf{I}=\mathbf{1} .
$$

The consistency condition reads

$$
\dot{F}\left(\boldsymbol{\sigma}, \sigma^{\mathrm{M}}, f\right)=\frac{\partial F}{\partial \sigma}: \dot{\boldsymbol{\sigma}}+\frac{\partial F}{\partial \sigma^{\mathrm{M}}} \dot{\sigma}^{\mathrm{M}}+\frac{\partial F}{\partial f} \dot{f}=0 .
$$

\section{EXTENSION TO THE GURSON-TVERGAARD MATERIAL MODEL}

This chapter introduces an extension to the Gurson-Tvergaard material model. The new constitutive equation is formulated using the basic laws, axioms and definitions of thermomechanics, and the results provided by damage mechanics. The main difference between the present model and that by GursonTvergaard lies in the description of elastic deformation and hardening.

The extended constitutive equation models the elastic deformation by taking into consideration the stiffness reduction of the material due to voids, whereas the original Gurson-Tvergaard formulation simply uses the Hooke's law. The difference between these two descriptions may appear to be negligible, since the elastic deformation plays a minor role in the total deformation of the body. It is true that elastic deformation is small by comparison with plastic deformation and therefore the additive deformation due to damage is negligible. The idea of introducing the "correct elastic stiffness" is that it enables one to introduce the methods of damage mechanics and thereby gives the necessary information to construct the yield function $F$.

The difference in the description of hardening is that the extended model replaces the hardening evolution equation (33) by Tvergaard with a damage mechanics description, where a similar effect is obtained by taking into account that the effective stress $\tilde{\boldsymbol{\sigma}}$, as described in Fig. 1, takes a higher value with growing porosity $f$. 


\subsection{Material model expressed by two functions}

The independent state variables of the extension to the Gurson-Tvergaard material model are the strain tensor $\boldsymbol{\varepsilon}$, the void-plastic strain tensor $\boldsymbol{\varepsilon}^{\mathrm{pv}}$, and the void volume fraction $f$. This means that by following the concept of the original model, the thermal effects are neglected. The extended Gurson-Tvergaard material model assumes that the state is expressed by the difference $\boldsymbol{\varepsilon}-\boldsymbol{\varepsilon}^{\mathrm{pv}}$. The state is expressed by the specific complementary Helmholtz free energy $\psi$ having the form of Eq. (6):

$$
\psi^{\mathrm{c}}(\boldsymbol{\sigma}, f)=\frac{1}{2 \rho}\left[\frac{1}{3(3 \lambda+2 \mu)}(1+A f)[\mathbf{1}: \boldsymbol{\sigma}]^{2}+\frac{1}{2 \mu}(1+B f) \mathbf{s}: \mathbf{s}\right] .
$$

The yield function $F$ is assumed to have the form

$$
\begin{aligned}
& F\left(\boldsymbol{\sigma}, g ; \boldsymbol{\varepsilon}^{\mathrm{e}}, f\right)=\left\{\frac{J_{\mathrm{vM}}(\boldsymbol{\sigma})}{J_{\mathrm{vM}}\left(\mathbf{C}: \boldsymbol{\varepsilon}^{\mathrm{e}}\right)}\right\}^{2}+2 f q^{1} \cosh \left(\frac{q^{2} \mathbf{1}: \boldsymbol{\sigma}}{2 J_{\mathrm{vM}}\left(\mathbf{C}: \boldsymbol{\varepsilon}^{\mathrm{e}}\right)}\right) \\
& -\left(1+q^{3} f^{2}\right)+3\langle 1-f\rangle \frac{\langle f\rangle q^{1} q^{2}}{J_{\mathrm{vM}}\left(\mathbf{C}: \boldsymbol{\varepsilon}^{\mathrm{e}}\right)} \sinh \left(\frac{q^{2} \mathbf{1}:\left(\mathbf{C}: \boldsymbol{\varepsilon}^{\mathrm{e}}\right)}{2 J_{\mathrm{vM}}\left(\mathbf{C}: \boldsymbol{\varepsilon}^{\mathrm{e}}\right)}\right) g .
\end{aligned}
$$

In Eq. (68) the notation $\boldsymbol{\varepsilon}^{\mathrm{e}}=\boldsymbol{\varepsilon}-\boldsymbol{\varepsilon}^{\mathrm{pv}}$ is introduced. The notation $\langle\cdot\rangle$ stands for the Macaulay brackets. They are adopted in Eq. (68) to guarantee that the value of the void volume fraction $f$ remains between 0 and 1 .

Using Eqs. (7a), (7b), (8), and (17), we obtain

$$
\begin{gathered}
\boldsymbol{\varepsilon}-\boldsymbol{\varepsilon}^{\mathrm{pv}}=\rho \frac{\partial \psi^{\mathrm{c}}(\boldsymbol{\sigma}, f)}{\partial \boldsymbol{\sigma}}=\frac{1}{3(3 \lambda+2 \mu)}(1+A f) \mathbf{1}: \boldsymbol{\sigma} \mathbf{1}+\frac{1}{2 \mu}(1+B f) \mathbf{s}, \\
g=\rho \frac{\partial \psi^{\mathrm{c}}(\boldsymbol{\sigma}, f)}{\partial f}=\frac{1}{2}\left[\frac{A}{3(3 \lambda+2 \mu)}[\mathbf{1}: \boldsymbol{\sigma}]^{2}+\frac{B}{2 \mu} \mathbf{s}: \mathbf{s}\right]
\end{gathered}
$$

State equation (69) exploits the fact that for the present material model the inelastic strain tensor $\boldsymbol{\varepsilon}^{1}$ is the void-plastic strain tensor $\boldsymbol{\varepsilon}^{\mathrm{pv}}$.

Following $\left[{ }^{8}\right]$, the normality rule takes the form

$$
\dot{\boldsymbol{\varepsilon}}^{\mathrm{pv}}=\stackrel{\circ}{\lambda} \frac{\partial F\left(\boldsymbol{\sigma}, g ; \boldsymbol{\varepsilon}^{\mathrm{e}}, f\right)}{\partial \boldsymbol{\sigma}},
$$

where

$$
\dot{f}=\stackrel{\circ}{\lambda} \frac{\partial F\left(\boldsymbol{\sigma}, g ; \boldsymbol{\varepsilon}^{\mathrm{e}}, f\right)}{\partial g},
$$

and $\stackrel{\circ}{\lambda}$ is the plastic multiplier. Substitution of Eq. (68) into (71a) gives 


$$
\dot{\boldsymbol{\varepsilon}}^{\mathrm{pv}}=\stackrel{\circ}{\lambda}\left\{\frac{3 \mathbf{s}}{\left[J_{\mathrm{vM}}\left(\mathbf{C}: \boldsymbol{\varepsilon}^{\mathrm{e}}\right)\right]^{2}}+\frac{f q^{1} q^{2}}{J_{\mathrm{vM}}\left(\mathbf{C}: \boldsymbol{\varepsilon}^{\mathrm{e}}\right)} \sinh \left(\frac{q^{2} \mathbf{1}: \boldsymbol{\sigma}}{2 J_{\mathrm{vM}}\left(\mathbf{C}: \boldsymbol{\varepsilon}^{\mathrm{e}}\right)}\right) \mathbf{1}\right\} .
$$

Derivation of Eq. (72) took into account the equalities

$$
\frac{\partial J_{\mathrm{vM}}(\boldsymbol{\sigma})}{\partial \boldsymbol{\sigma}}=\frac{3}{2} \frac{\mathbf{s}}{J_{\mathrm{vM}}(\boldsymbol{\sigma})}, \quad \frac{\partial(\mathbf{1}: \boldsymbol{\sigma})}{\partial \boldsymbol{\sigma}}=\mathbf{1}: \frac{\partial \boldsymbol{\sigma}}{\partial \boldsymbol{\sigma}}=\mathbf{1}: \mathbf{I}=\mathbf{1} .
$$

Using Eq. (18b), Eq. (72) can be written as

$$
\dot{\boldsymbol{\varepsilon}}^{\mathrm{pv}}=\stackrel{\circ}{\lambda}\left\{\frac{3 \mathbf{s}}{\left[J_{\mathrm{vM}}(\tilde{\boldsymbol{\sigma}})\right]^{2}}+\frac{f q^{1} q^{2}}{J_{\mathrm{vM}}(\tilde{\boldsymbol{\sigma}})} \sinh \left(\frac{q^{2} \mathbf{1}: \boldsymbol{\sigma}}{2 J_{\mathrm{vM}}(\tilde{\boldsymbol{\sigma}})}\right) \mathbf{1}\right\} .
$$

The first invariant of the void-plastic strain rate takes the form

$$
\mathbf{1}: \dot{\boldsymbol{\varepsilon}}^{\mathrm{pv}}=\stackrel{\lambda}{\lambda} \frac{f q^{1} q^{2}}{J_{\mathrm{vM}}(\tilde{\boldsymbol{\sigma}})} \sinh \left(\frac{q^{2} \mathbf{1}: \boldsymbol{\sigma}}{2 J_{\mathrm{vM}}(\tilde{\boldsymbol{\sigma}})}\right),
$$

where we have taken into account that $\mathbf{1}: \mathbf{s}=0$ and $\mathbf{1}: \mathbf{1}=3$.

Substituting Eq. (68) into (71b) gives

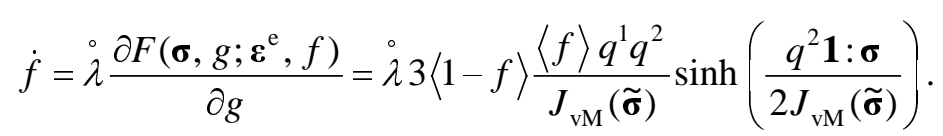

Comparison of Eqs. (75) and (76) gives

$$
\dot{f}=\langle 1-f\rangle \frac{\langle f\rangle}{f} \mathbf{1}: \dot{\boldsymbol{\varepsilon}}^{\mathrm{pv}} .
$$

Evolution equation (77) is similar to Eq. (42). Furthermore, it extends the meaning of Eq. (42) by guaranteeing that the value of the void volume fraction $f$ cannot exceed unity or take negative values. The effective stress tensor $\tilde{\boldsymbol{\sigma}}$ is obtained from Eq. (20).

The thermomechanical theory of plasticity adopts consistency condition for determination of the value of the plasticity multiplier $\lambda$. It is

$$
\dot{F}=0 .
$$

Applying consistency condition (78) to yield function (68) reveals

$$
\dot{F}\left(\boldsymbol{\sigma}, g ; \boldsymbol{\varepsilon}^{\mathrm{e}}, f\right)=\frac{\partial F}{\partial \sigma}: \dot{\boldsymbol{\sigma}}+\frac{\partial F}{\partial g} \dot{g}+\frac{\partial F}{\boldsymbol{\varepsilon}^{\mathrm{e}}}: \dot{\boldsymbol{\varepsilon}}^{\mathrm{e}}+\frac{\partial F}{\partial f} \dot{f}=0,
$$

where the chain rule is used. 


\subsection{Clausius-Duhem inequality}

According to Santaoja $\left[{ }^{8}\right]$, the Clausius-Duhem inequality takes the following form:

$$
\boldsymbol{\sigma}: \dot{\boldsymbol{\varepsilon}}^{\mathrm{pv}}+g \dot{f} \geq 0
$$

Using Eq. (74), we obtain

$$
\boldsymbol{\sigma}: \dot{\boldsymbol{\varepsilon}}^{\mathrm{pv}}=\stackrel{\circ}{\lambda}\left[\frac{3 \boldsymbol{\sigma}: \boldsymbol{\sigma}}{\left[J_{\mathrm{vM}}(\tilde{\boldsymbol{\sigma}})\right]^{2}}+\frac{f q^{1} q^{2}}{J_{\mathrm{vM}}(\tilde{\boldsymbol{\sigma}})} \sinh \left(\frac{q^{2} \mathbf{1}: \boldsymbol{\sigma}}{2 J_{\mathrm{vM}}(\tilde{\boldsymbol{\sigma}})}\right) \mathbf{1}: \boldsymbol{\sigma}\right],
$$

where the fact that $\mathbf{s : \sigma = \sigma : \sigma}$ is taken into account. Since the plasticity multiplier $\lambda$ is always non-negative, the expression between the square brackets has to be non-negative. The first term of Eq. (81) is always non-negative. Since the quantities $\left(f, q^{1}, q^{2}, J_{\mathrm{vM}}(\tilde{\boldsymbol{\sigma}})\right)$ are non-negative, the second term of Eq. (81) is also non-negative. Thus the first term of Clausius-Duhem inequality (81) is always non-negative.

Equation (17) shows that the force $g$ is always non-negative. As Eq. (76) indicates, rate $f$ takes a negative value when 1: $\boldsymbol{\sigma}$ is negative. This implies that there are states when the term $g \dot{f}$ takes a negative value, and therefore the Clausius-Duhem inequality (80) is not satisfied. The following section discusses how to prevent this problem.

\section{COMPARISON OF THE GURSON-TVERGAARD MATERIAL MODEL WITH THE EXTENDED ONE}

The value of the plasticity multiplier $\stackrel{\circ}{\lambda}$ in Eq. (63) is obtained by applying the consistency condition. For the Gurson-Tvergaard material model it is given by Eq. (66). The evolution equation for the hardening quantity $\sigma^{\mathrm{M}}$ is given by Eq. (33). The corresponding expressions for the extended material model are given by Eqs. (74), (78), and (80).

Comparison of Eqs. (64) and (74) shows that the "fundamental parts" of the expressions for the void-plastic strain rate tensor $\dot{\boldsymbol{\varepsilon}}^{\mathrm{pv}}$ differ only in the description of "hardening". The hardening quantity $\sigma^{\mathrm{M}}$ in Eq. (64) is replaced in the extended model by $J_{\mathrm{vM}}(\tilde{\boldsymbol{\sigma}})$. According to Tvergaard $\left[{ }^{2}\right], \sigma^{\mathrm{M}}$ is the equivalent (in the sense of von Mises) to tensile flow stress in the matrix material, disregarding local stress variations. Based on damage mechanics, the effective stress $\tilde{\sigma}$ gives the value of the stress in the matrix material (disregarding local stress variations). This implies that

$$
\sigma^{\mathrm{M}}=J_{\mathrm{vM}}(\tilde{\boldsymbol{\sigma}}) .
$$


Naturally, the values of these two quantities are obtained by totally different equations, as Eqs. (33) and (20) show. Also the plasticity multipliers $\grave{\lambda}$ for these two models are different as is seen from Eqs. (66) and (79).

Evolution equations for the void volume fraction $f$ are given by Eqs. (54) and (77). Comparison of Eqs. (54) and (77) shows that for positive $f$ they coincide. However, the extended material model does not allow unrealistic values for the void volume fraction $f$ to force it to take values between 0 and 1 .

\section{DISCUSSION AND CONCLUSIONS}

The idea of the present work was to evaluate the foundations of the GursonTvergaard material model and to derive the constitutive equation by using thermomechanics and damage mechanics. Based on the evaluation, some criticism was expressed and an extension to the Gurson-Tvergaard material model was derived. In the following, the criticism and the basic concepts of the extended Gurson-Tvergaard material model are outlined.

Gurson $\left.{ }^{1}\right]$ derived yield function $F$ for materials having different types of voids in an elastic-perfectly plastic matrix material. Gurson used the upper bound theorem in the derivation of yield functions $F$. Today a special case, where the voids are spherical and the matrix material is fully plastic, is referred to as the Gurson model.

Tvergaard $\left[{ }^{2}\right]$ modified the Gurson model by including in the model elastic deformation and hardening of the matrix material. He also added three material parameters into the constitutive equation. These material parameters are denoted by $q^{1}, q^{2}$, and $q^{3}$. The model proposed by Tvergaard has three internal variables: the void-plastic strain tensor $\boldsymbol{\varepsilon}^{\mathrm{pv}}$, the void volume fraction (porosity) $f$, and the equivalent tensile flow stress in the matrix material $\sigma^{\mathrm{M}}$. By the void-plastic strain tensor $\varepsilon^{\mathrm{pv}}$ is meant a strain that combines the strain due to plastic yield of the matrix material and void nucleation and growth. Tvergaard called $\varepsilon^{\mathrm{pv}}$ the plastic strain and denoted it by $\varepsilon^{\mathrm{p}}$. The evolution equations given by Tvergaard for the void volume fraction $f$ and the void plastic strain tensor $\boldsymbol{\varepsilon}^{\mathrm{pv}}$ are acceptable. On the other hand, the evolution equation for the equivalent tensile flow stress in the matrix material $\sigma^{\mathrm{M}}$ arouse discussion. Tvergaard replaced the constant $\sigma^{\mathrm{Y}}$ with the variable $\sigma^{\mathrm{M}}$. Since the role of the constant $\sigma^{\mathrm{Y}}$ in the Gurson model was to make some quantities dimensionless, the replacement of the constant $\sigma^{\mathrm{Y}}$ by a hardening variable $\sigma^{\mathrm{M}}$ does not have any physical justification. Furthermore, the upper bound approach used by Gurson is not valid for the strain hardening case. Also the introduction of the three material parameters $q^{1}, q^{2}$, and $q^{3}$ needs physical explanation.

Chu and Needleman $\left[{ }^{3}\right]$ and Tvergaard and Needleman $\left[{ }^{4}\right]$ added extra terms to the void volume fraction $f$. These extra terms violate the axiom of conservation of mass and therefore have to be excluded. 
The terminology related to the Gurson-Tvergaard material model has not been established. When referring to the Gurson-Tvergaard material model some writers mean the model introduced by Gurson $\left[{ }^{1}\right]$ and modified by Tvergaard $\left[{ }^{2}\right]$. However, other writers include in the Gurson-Tvergaard model also the modifications by Chu and Needleman $\left[{ }^{3}\right]$ and Tvergaard and Needleman $\left[{ }^{4}\right]$, or one of them.

The present work tried to formulate the Gurson-Tvergaard material model by using thermomechanics and damage mechanics. The object of the analysis was the constitutive equation by Gurson $\left[{ }^{1}\right]$ and Tvergaard $\left[{ }^{2}\right]$. The obtained new material model is here called the extended Gurson-Tvergaard material model.

The yield function $F$ proposed by Gurson $\left[{ }^{1}\right]$ and modified by Tvergaard $\left[{ }^{2}\right]$ was adopted almost in its original form into the extended Gurson-Tvergaard material model. Two major changes, however, were made. First, an "extra" term to the yield function $F$ was added to fulfil the requirements set by the evolution equation for the void volume fraction $f$. The extended model neglected (as did Tvergaard) the influence of the elastic response of the matrix on the evolution equation for the void volume fraction $f$. This was done, because the elastic response of the matrix has a minimal influence on the evolution of the void volume fraction $f$. Second, the quantity $\sigma^{\mathrm{M}}$, introduced by Tvergaard, was replaced by the von Mises value of the effective stress $J_{\mathrm{vM}}(\tilde{\boldsymbol{\sigma}})$. The introduction of the effective stress tensor $\tilde{\boldsymbol{\sigma}}$ fits well the thermodynamical approach and does not require any special evolution equation, since damage mechanics gives an expression for it. According to Tvergaard $\left[{ }^{2}\right], \sigma^{\mathrm{M}}$ is the equivalent (in the sense of von Mises) tensile flow stress in the matrix material, disregarding local stress variations. Based on damage mechanics, the effective stress $\tilde{\boldsymbol{\sigma}}$ gives the value of the stress in the matrix material (disregarding local stress variations). This implies that $\sigma^{\mathrm{M}}=J_{\mathrm{vM}}(\tilde{\boldsymbol{\sigma}})$. Also, the plasticity multiplier $\lambda$ makes a difference between the Gurson-Tvergaard material model and the extended Gurson-Tvergaard material model.

Thermomechanical evaluation allows to prove that the material model fulfils the requirements set by thermomechanics. The key role is the satisfaction of the Clausius-Duhem inequality. The standard procedure of this consideration is to combine the basic laws and axioms of thermomechanics and to interpret the resulting expression as the Clausius-Duhem inequality. Section 5.2 showed that when the hydrostatic part of the stress is tension, the extended Gurson-Tvergaard material model always satisfies the Clausius-Duhem inequality. In compression, however, there are states which are not allowed. This means that if this constitutive equation is used, e.g. in finite element code, the program must also perform verification of the satisfaction of the Clausius-Duhem inequality.

As a conclusion, the author wants to underline that the above criticism was based on pure scientific analysis. Despite its many weaknesses, the GursonTvergaard material model might be suitable for engineering purposes. This follows from the fact that anything better simply does not exist. 


\section{ACKNOWLEDGEMENTS}

This publication was prepared during my work as a senior assistant of the Laboratory for Mechanics of Materials at Helsinki University of Technology (HUT). I wish to thank the Head of the Laboratory, Professor Mauri Määttänen, for enabling me to concentrate on writing this paper. I am grateful to Professor Martti Mikkola for discussions and valuable comments.

\section{REFERENCES}

1. Gurson, A. L. Continuum theory of ductile rupture by void nucleation and growth. Part I: Yield criteria and flow rules for porous ductile media. J. Eng. Mater. Technol., Trans. ASME, 1977, 99, 2-15.

2. Tvergaard, V. Influence of voids on shear band instabilities under plane strain conditions. Int. J. Fract., 1981, 17, 389-407.

3. Chu, C. C. and Needleman, A. Void nucleation effects in biaxially stretched sheets. J. Eng. Mater. Technol., Trans. ASME, 1980, 102, 249-256.

4. Tvergaard, V. and Needleman, A. Analysis of the cup-cone fracture in a round tensile bar. Acta Metall., 1984, 32, 157-169.

5. Eshelby, J. D. The determination of the elastic field of an ellipsoidal inclusion, and related problems. Proc. R. Soc. Lond. A, 1957, 241, 376-396.

6. Mackenzie, J. K. The elastic constants of a solid containing spherical holes. Proc. R. Soc. Lond. $B, 1950,63,2-11$.

7. Malvern, L. E. Introduction to the Mechanics of a Continuous Medium. Prentice-Hall, Englewood Cliffs, New Jersey, 1969.

8. Santaoja, K. Lecture Notes on Thermomechanics. Helsinki University of Technology, Laboratory for Mechanics of Materials, Research Reports, TKK-Lo-32, 2001.

9. Chaboche, J.-L. Description thermodynamique et phénoménologique de la viscoplasticité cyclique avec endommagement. Office National d'Etudes et de Recherches Aérospatiales. Chatillon, Publication No. 1978-3. 1978.

10. Lemaitre, J. and Chaboche, J.-L. Mechanics of Solid Materials. Cambridge University Press, New York, 1990.

11. Rabotnov, Y. N. Creep rupture. In Proc. XIIth International Conference on Applied Mechanics (Hetényi, M. and Vincenti, W. G., eds.). Springer-Verlag, Berlin, 1968, 342-349.

12. Hult, J. Creep in continua and structures. In Topics in Applied Continuum Mechanics (Zeman, J. L. and Ziegler, F., eds.). Springer-Verlag, Vienna, 1974, 137-155.

13. Santaoja, K. Continuum damage mechanics approach to describe the multidirectional microcraking of ice. In Proc. 8th Offshore Mechanics and Arctic Engineering Symposium (Sinha, N. K., Sodhi, D. S., and Chung, J., eds.). The American Society of Mechanical Engineers, Hague, 1989, vol. IV, 55-65.

14. Santaoja, K. Mathematical Modelling of Deformation Mechanisms in Ice. Doctoral Thesis. Helsinki University of Technology. The Technical Research Centre of Finland, Espoo, Finland, VTT Research Report 676, 1990.

15. Santaoja, K. Viscous and Elastic-Plastic Material Model in the ABAQUS. The Technical Research Centre of Finland, Espoo, Finland, VTT Publication 144. 1993.

16. Zienkiewicz, O. C. The Finite Element Method, 3rd ed. McGraw-Hill, Berkshire, 1977.

17. Kachanov, L. M. Fundamentals of the Theory of Plasticity. Mir, Moscow, 1974.

18. Narasimhan, M. N. L. Principles of Continuum Mechanics. J. Wiley, New York, 1993.

19. Lubliner, J. Plasticity Theory. Macmillan, Singapore, 1990. 


\title{
Gursoni-Tvergaardi mudeli hindamine, kasutades purunemise mehaanikat ja termodünaamikat
}

\author{
Kari Santaoja \\ Artiklis on tuletatud materjali käitumist kirjeldava Gursoni-Tvergaardi \\ mudeli uus kuju, mis rahuldab alati Clausiuse-Duhemi võrratust.
}

